\title{
ESCRITORES EN LA PRENSA: AUTORÍA Y AUTORIDAD*
}

\section{Jaime Galgani Muñoz**}

\section{Resumen}

El presente artículo se detiene en la relación existente entre escritores y prensa y el fundamento que sustenta los principios de legitimación autorial que permiten su presencia en el periodismo moderno a partir del siglo XIX hasta nuestros días. Acogiendo la hipótesis de que, a la base de la mediación entre autor y lector, se encuentra el concepto de ethos retórico, se presentan procesos y escritores que, con su trabajo, dan cuenta de que, tratándose de la prensa de opinión (columnas, artículos, crónicas, etc.), la relación entre retórica y ética es fundamental.

Palabras clave: ethos retórico, periodismo moderno, periodismo de opinión, columna cultural.

\section{WRITERS IN THE PRESS: AUTHORSHIP AND AUTHORITY}

\begin{abstract}
This article examines the relationship between writers and the press as well as the groundwork that supports the principles of legitimization of authorship and allows its presence in modern journalism since the nineteenth century. Accepting the hypothesis that rhetorical ethos is the basis for mediation between author and reader, this article presents processes and writers that acknowledge through their work that since the press is about opinion (columns, articles, chronicles, etc.), the relationship between rhetoric and ethics is fundamental.
\end{abstract}

Keywords: rhetorical ethos, modern journalism, opinion journalism, cultural column.

Proyecto de investigación Fondecyt Regular No. 1160222: Literatura y Periodismo: corresponsales y columnistas de guerra chilenos (1879-1945).

* Doctor en Literatura. Académico Universidad Metropolitana de Ciencias de la Educación, Santiago, Chile. jaime.galgani@umce.cl 


\section{El ethos retórico, condición de mediación entre el público y el escritor en la prensa}

La columna de opinión, en sus diversas variantes, se hace valorar por una autoridad fundada previamente por el protagonismo del autor en alguna actividad cultural previa; en nuestro caso, por el reconocimiento del escritor como autor de obras literarias reconocidas. El peso cultural de su autor es tan relevante que, por ese motivo, la columna de opinión es buscada y leída. En este sentido, es un género tal que, habiendo nacido al mismo tiempo que el periodismo «objetivo», vinculado a la noticia y al reportaje, goza precisamente de destacar por la subjetividad autorial. No deja de resultar paradójico que su desarrollo se haya dado precisamente al mismo tiempo que, a la validez de todas las disciplinas (incluyendo el periodismo, por cierto), se le exigiera el correlato de la «evidencia», como si el periodismo antiguo, el de la primera parte del siglo XIX, que incorporaba en un solo cuerpo objetividad y autoridad personal, se hubiera separado en dos, dejando para la noticia el rigor de la primera y, para el artículo o columna, el subjetivismo de la opinión.

En el momento en que el escritor ingresa por algún medio en el periodismo, debe considerar que arriesga el prestigio ganado con sus obras. Su participación en ellas puede reafirmar la estima que ha obtenido de su público lector como también la puede dañar, pues la misma subjetividad que allega para sí el autor es la que reclaman quienes lo siguen, tanto para aceptarlo como para rechazarlo. De eso supo bien Emile Zola cuando publicó su declaración sobre el caso Dreyfus; el título mismo de dicha pronunciación, "J'accuse» (L'Aurore, 13 de enero de 1898), revela el servicio que puede hacer la autoridad representada por un capital específico y social en beneficio de una causa determinada. Hay algunos que escriben para prestigiarse, otros para comer, otros para arrimarse a algún escaño político; Zola lo hizo en función de la irritación que le producía la injusticia y de su necesidad de «Mettre en marche la verité», a fin de que la verdad que muchos transan por un plato de lentejas fuera el presupuesto incuestionable de su primogenitura cultural en el escenario de los escritores naturalistas franceses. El medio que escogió para expresarse fue la prensa, que, como dijo José Martí, se constituyó a fines del siglo XIX en «el nuevo espacio para las ideas» (Prólogo a Poema del Niágara de Juan Antonio Pérez Bonalde, 1882). Así también lo hizo Darío en Latinoamérica y Unamuno en España (por ejemplo 80 artículos 
en la prensa de Salamanca entre 1891 y 1902), tal como todo escritor que viera en la prensa -ese nuevo espacio moderno de interacción socialuna necesaria continuidad con su obra literaria.

La presencia de escritores en la prensa ha seguido los avatares del periodismo. Primero fue el escritor ilustrado que publicaba tanto editoriales como crónicas, comentarios, críticas de arte, todo... porque él era todo, porque era simplemente al mismo tiempo un intelectual, un político, un jurista, un entendido en querellas, en política y en religión. Después, por la vía de la modernización del periodismo y de la especialización profesional, el letrado decimonónico debió abandonar la oficina de dirección para dar lugar a los jóvenes burócratas de la noticia y a la objetividad como norma, propuesta, derrotero y fantasma positivista, generado para ofrecer la ilusión de realidad que produce el contacto con la novedad inmediata. Al viejo escritor le dieron un lugar en la sala de redacción o le permitieron derechamente que escribiera sus artículos y columnas en casa; desde ahora, la única normatividad que dirigiría su participación en el periódico estaría determinada por la libre inspiración que, tras el acontecer vertiginoso de la modernidad que hace que, a diferencia de los tiempos antiguos, cada día el ser humano amanezca con un problema y se acueste con otro (Martí), y sobre esa base dé luz a las pequeñas «obras fúlgidas» (Martí, Prólogo al Poema del Niágara) que son sus columnas o artículos breves, inspirados, estructurados con el impulso que tiene una onda que, disparando una certera pedrada sobre el aire, en un solo vuelo pretende derrotar al gigante Goliat por medio de sus ironías, paradojas, retruécanos y alegorías. Décadas después, con el progreso incuestionable de los medios de comunicación y con el advenimiento de nuevos protagonistas sociales, el escritor debió achicar más aún su espacio en la sala de redacción para compartirlo con sociólogos, psicólogos, periodistas titulados a distancia, tarotistas y tertulianos. Para que no se pelearan tanto personalidades tan disímiles, la misma tecnología les proporcionó los medios para no verse directamente y, así, escribir sus columnas ya fuera en casa como en un hotel, en un tren entre Ginebra y París o, incluso, disfrutando de unas deliciosas vacaciones en un resort all inclusive. Como sea, al escritor, antiguo señor feudal de la imprenta y de todas sus posesiones añejas, le han quitado indoloramente sus últimas colonias en la prensa y no puede participar más que como un allegado. No es cosa de lamentar, por cierto, pues el periodismo necesita 
enriquecerse de múltiples miradas y es justo que pida y se sirva de lo que cada cual hace y gusta de hacer bien: al economista, proyecciones y tendencias; al sociólogo, migraciones y desplazamientos; al tarotista, futuro y presente desconocidos; al tertuliano, amores y desamores de famosos; y al escritor, lo único que le queda y que es preferentemente lo suyo, es decir, estilo, argumentación retórica, disuasión y vuelo literario.

Sin embargo, lo que este artículo se propone revisar consiste en la consideración de que el capital autorial que el escritor pone en juego al ingresar en la prensa, tanto antes como ahora, está mediado por una consideración fundamental; a saber, el vínculo que hay entre retórica y ética. Los recursos literarios y retóricos son una condición necesaria pero no suficiente para lograr adhesión y seguimiento cuando se trata de la prensa de opinión, puesto que lo que produce comunicación es el asentimiento que un determinado tipo de lectores conceda a la verdad que expresa un columnista y la coherencia que, en dicha materia, pueda demostrar. Es cierto que, en algunos géneros, la consecuencia ética puede ser menos relevante (como la crónica de viajes, por ejemplo), pero, a medida que las temáticas específicamente no literarias exigen mayor compromiso con una postura ideológica, entonces la cuestión ética se hace más relevante. En definitiva, lo que diferencia los géneros positivistas del periodismo (como el reportaje y la noticia) de los géneros de opinión está en que aquellos se apoyan en la "prueba», mientras que éstos lo hacen en el testimonio autorial acompañado de los recursos y el estilo persuasivo de una pluma certeramente asertiva.

Ahora bien, sin entrar en la difícil clasificación de géneros a través de los cuales se hace presente el escritor en la prensa (artículo, columna, crónica, etc.), lo que interesa fundamentalmente es ver cuál es, en términos generales, el dispositivo mediador entre él y su público y cómo éste se constituye en el elemento unificador, y de alguna manera, identificador, de los diversos tipos de presencia del escritor en la prensa. La propuesta de Fernando López Pan (2005) es que dicho elemento unificador es «el concepto de ethos retórico (carácter/talante/imagen intratextual)» (12).

Pienso que esa noción ampara las enumeraciones -inevitablemente incompletas- que pretenden describir todas las posibilidades del género; ahonda en los habituales rasgos de firma, periodicidad fija y libertad expresiva y temática; y actúa como estrategia retórica configuradora de la columna. (12-13) 
Para demostrar su tesis, López Pan (2005) acude a la expresión de Aristóteles cuando habla de la "prueba retórica basada en el ethos»: «Cuando el discurso se dice de tal manera que hace digno de fe al que lo dice, pues a las personas decentes las creemos más y antes...» (ctd. en López Pan 12), constituyéndose en lo que, para el filósofo es la prueba más importante, razón por la cual es común ver que, frecuentemente, para refutar la argumentación del articulista, se recurre al argumento ad hominem, es decir, al desmoronamiento de la validez moral del autor para pronunciarse sobre tal o cual aspecto (13). Se asume, entonces, que la cuestión retórica (es decir, la palabra ingeniosa y artísticamente bien enhebrada) debe convertirse en palabra confiable. Ejemplo de ello son las polémicas que, de tanto en tanto, se dan sobre el valor que puede tener la palabra de algunos escritores para hablar sobre determinadas materias (v.gr.: Günter Grass cuando reconoció en su biografía (2007) haber pertenecido a las Waffen-SS).

López Pan, sin desconocer otros componentes válidos, concluye su presentación expresando que

La columna -sea interpretativa/de análisis, de opinión o literaria-, dado el sello personal que la caracteriza, es un género privilegiado dentro del periódico para la expresión del ethos [...]. Sobre él, sobre esa impronta textual del autor que se perfila con el sucederse de los textos, se asienta la fuerza persuasiva de la columna. $\mathrm{Y}$ dado el contexto retórico mediado, más que una adaptación del autor a los lectores, son éstos quienes descubren en las páginas de los periódicos alguien con quien sintonizan y de quien se fían; alguien con el que comparten, en el pequeño universo de un texto, la misma mirada sobre el mundo y la vida. (14)

\section{De la ciudad modernizada a la ciudad politizada: una mirada de contexto para Hispanoamérica}

Releer las páginas de La ciudad letrada (1998) de Ángel Rama permite comprender el contexto en que se enmarca el surgimiento del periodismo moderno en la segunda mitad del siglo XIX y la evolución que ha experimentado en buena parte del siglo XX. En los capítulos titulados «La ciudad modernizada» $\mathrm{y}$ «La ciudad se politiza», se advierte 
la transición que ha vivido la ciudad latinoamericana en dos períodos claramente identificables, probablemente los que con mayor nitidez han contribuido a conformar el perfil de estas urbes que, en su esquizoide devenir, han configurado una identidad compleja y multivariada que hemos llegado a pensar como parte de su naturaleza. En estos períodos, relativamente similares en la mayor parte de nuestras naciones, también cambia la relación entre el escritor y el periodismo, que, aunque estrecha, se ve influenciada por las distintas temáticas que dominan la agenda política, cultural y social.

En el contexto de la ciudad modernizada, Rama (1998) postula que, aun teniendo en cuenta el desarrollo de una cierta promoción de los sectores mesocráticos de la sociedad, de la llegada de inmigrantes a los grandes centros urbanos, de las posibilidades que brindaban nuevos horizontes proporcionados por la Educación, no se dio en la América Hispana el «reconocimiento del esfuerzo individual» que, en los Estados Unidos, llegó a configurar el mito del self-made man, mito que «[e]n el campo letrado, proveyó de dos figuras heroicas y solitarias: el periodista y el abogado» (64). Estas figuras cultivan el imaginario de un tipo de profesional que, en resguardo de la justicia y del derecho, ofrece un contrapeso significativo al poder feudatario que en Norteamérica ostentaba extraordinarias cuotas de «concentración del poder» (65).

Ese periodista que escribe en un pequeño diario pueblerino, en el cual denuncia las injusticias y las arbitrariedades de los poderosos a los que concluye venciendo y ese abogado pobre que ante los tribunales vence las maquiavélicas conjuras de los ricos y restablece los derechos a la inocencia del acusado, son mitos urbanos y letrados que no se desarrollaron en América Latina. (65)

Sabemos que ese imaginario norteamericano, que se ha hecho extensivo hasta la literatura y el cine actuales, ha levantado la imagen del justiciero solitario, de abogados, periodistas, superhéroes, e incluso la mezcla entre algunos de ellos (Superman es un caso). Pero este imaginario se contradice con el «enorme peso de las instituciones latinoamericanas que configuran el poder y [...] la escasísima capacidad de los individuos para enfrentarlas y vencerlas» (Rama 65). Este horizonte ha hecho que «la sociedad urbana latinoamericana oper[e] dentro de modelos más 
colectivizados» y que «sus mitos opositores del poder pas[e]n a través de la configuración de grupos, de espontáneas coincidencias protestatarias, de manifestaciones y reclamaciones multitudinarias» (65). La literatura latinoamericana abunda en ejemplos tanto de la impotencia en la lucha por la justicia frente al poder real (v.gr. El mundo es ancho y ajeno (1941) de Ciro Alegría), como de las asonadas violentas de manifestaciones colectivas (v.gr. Los de abajo (1915), de Mariano Azuela).

Sin embargo, por lo que respecta a la ciudad moderna, es decir, a aquella composición urbana que se establece entre 1870 y 1900 aproximadamente, la actividad intelectual de los escritores, crecientemente ubicados en un campo literario más autónomo, ofrece el caso del escritor-columnista, escritor-cronista que ve en el periódico un espacio de participación en la vida social, desplegando, gracias a sus estrategias discursivas (como se ha visto en ejemplos citados anteriormente), una suerte de crítica a la modernidad y a los procesos que ella contiene. Un caso interesante, también comentado por Rama, es el de Justo Sierra Méndez (1848-1912). Además de escritor, fue historiador, periodista, poeta y político mexicano. Su participación en el periódico La libertad le permitió plantear su crítica al devenir político de su país y, desde ahí, instalarse como un actor importante en sus procesos de renovación. Ejemplo interesante también es el caso de Francisco García Cisneros (1877- ?), un escritor y periodista cubano que

[f] undó y dirigió la revista «Gris» $\mathrm{y}$ «Azul» y fue redactor de «La Habana Elegante» y «El Hogar». Corresponsal y amigo de gran número de escritores cubanos y del resto de América afiliados al Modernismo, defendió y propagó las ideas de este movimiento» [...] se radicó en Nueva York, donde tuvo a su cargo la «Crónica de Cuba« y «América» y colaboró en «Patria», «Revista de Cayo Hueso», «Las Tres Américas» y otras publicaciones en español. Más tarde se trasladó a Europa, desde donde continuó enviando a la prensa cubana sus crónicas, artículos, críticas y cuentos, que nunca recogió en libro. Colaboró en «El Fígaro« -en el que publicó más de cien trabajos-, «La Discusión», «Gráfico«, «Social», «Chic». (La prosa modernista párr. 2 )

García Cisneros, no suficientemente estudiado por la crítica académica, constituye un referente principal para entender las relaciones 
entre el campo literario y la prensa. En donde se consulten periódicos y revistas de comienzos del siglo XX es posible encontrar alguna crónica suya. Su postura, ligada a la estética modernista, quizás fue menos recogida por su oposición a Rodó y por su alineamiento con un proyecto que, en medio de las contingencias latinoamericanas, resultaba menos atractivo.

En Chile, escritores como Joaquín Díaz Garcés, Luis Orrego Luco y Augusto D'Halmar, se entretienen en deslizar a través de sus crónicas su descontento con el proceso modernizador. Suelen lamentar la pérdida de valores que representaba la antigua clase dirigente, critican el auge de la especulación financiera por sobre el crecimiento basado en la producción tradicional, destacan las diferencias sociales que la explotación del salitre y del carbón han establecido en el país, sospechan o se entusiasman alternativamente por las novedades de la modernización, dialogan con la noticia cotidiana, los escándalos económicos o sociales, los espantos de la gran guerra, el advenimiento de la cultura de masas, el quiebre de la bolsa de Walt Street, etc.

En las primeras décadas del siglo XX, la ciudad modernizada cede a «la ciudad politizada». Dicho cambio de modelo comienza a operarse en torno a las celebraciones del primer centenario de nuestras independencias. En Chile, Bernardo Subercaseaux ha analizado el período mostrando cómo, desde las capas intelectuales, especialmente desde los escritores, se materializa una crítica concreta al modelo de desarrollo imperante, dando evidencia de sus grietas y matices (Cfr. Genealogía de la vanguardia en Chile), Ángel Rama (1998), por su parte, plantea cómo, desde la revolución mexicana de 1911 hasta 1973 (Golpe de Estado en Chile), se dio un panorama de discusión activo en donde confluyeron diversos programas de conducción política en el subcontinente americano.

Tras la base engendradora de la modernidad latinoamericana (1870-1900), donde siempre podremos recuperar in nuce los temas, problemas y desafíos que animarán la vida contemporánea del continente, el siglo XX transcurre con agitación y movilidad creciente, como de crisis en crisis, primero en el periodo nacionalista que, incubado desde la apertura de la centuria, concluye triunfando de 1911 a 1930; luego en el populista que lo prolonga de 1930 a 1973 y que ya nos parece teleguiado por fuerzas internacionales ajenas 
a las voluntades latinoamericanas que sólo pueden oponerle respuestas, muchas veces confusas o errátiles, como si en otras circunstancias se recuperaran situaciones de los primeros siglos coloniales; por último el catastrófico período que se abre hacia 1973 y que sólo diez años después ha desvelado su insostenible gravedad. (83-84)

En este contexto, los escritores latinoamericanos asumen, según Rama, «en reemplazo de los sacerdotes, la conducción espiritual». «Médicos que se aplican al espíritu, por lo tanto los nuevos sacerdotes de la humanidad» (87). Deduzco que los instrumentos estilísticos que usan, «como la oratoria mayestática» (87) a la que alude Rama, manifiestos incluso en proyectos poéticos, son los que aplican también a sus colaboraciones en crónicas periodísticas, medio expresivo que se transforma en vital para su magisterio ideológico. Sin embargo, hay en sus colaboraciones a la prensa algo más que ideología, y es lo que llamamos «estilo», que no es otra cosa que la marca autorial que los hace presentes como escritores, una suerte de vuelo retórico que los hace, de alguna manera, por el recurso de la ironía y la crítica, manifestar su interés por la cuestión contingente sin dejarse arrebatar o subsumir por ella.

En este periodo, se destacan nombres que harían interminable este artículo. Para reflejar, sin embargo, el clima epocal y el peso que un escritor puede llegar a tener en el medio periodístico a través de sus crónicas de opinión, me parece interesante destacar el rol que ocupó el destacado novelista chileno, Joaquín Edwards Bello, quien puso al servicio del espíritu crítico nacional todo su capital autorial. Sus abundantes crónicas cumplen con las características del autor moderno y, sin entrar tan ávidamente en la arena política como en algún momento lo hicieron otros de su generación, concurren hábilmente a perfilar el proyecto nacional de aquellas décadas y el caldo de cultivo social en que se fecundaron, desarrollaron y manifestaron las grandes contiendas del siglo XX chileno.

Joaquín Edwards Bello, autor de La chica del Crillón (1935) y El roto (1920), escribió en torno a 12000 crónicas en la prensa. No hubo materia que le fuera ajena y produjo, durante varios años, una adhesión y seguimiento insuperables, «[e]n palabras de Alone, contra todos y a pesar de todos, Edwards bello se hace leer y discutir apasionadamente. Existe con innegable vigor, posee en el estilo [...] esa potencia eléctrica 
que no permite leer en frío» (Matus párr. 23). Sin duda, él es una clara demostración de un ethos retórico que reúne con maestría las cualidades literarias y éticas que otorgaban a su palabra la justa aprobación de «verdaderas» por cuanto, siguiendo la vieja escuela de los maestros de la verdad, Tolstoy, Zola, Daudet, en los cuales se formó la tradición literaria chilena del ‘900, se preocupaba de denunciar sin ambages las miserias del carácter nacional:

Una sutil aspereza se colaba entre tanto personaje que hacía aparecer y desaparecer de sus crónicas. Alérgico a cualquier eufemismo y siempre desenvuelto, el autor diseccionaba los valores y hábitos del país, llegando a decir, por ejemplo, que el chileno tenía la alegría del incendio, de la demolición, del velorio. Denunciaba a los lateros, esos que se ponen a divagar sobre la eutanasia o el comunismo, se reía de la vocación litigante del chileno (El pleito es una alegría para siempre); y describía con singular franqueza su propia naturaleza: A mí no me gusta que me lean. Parece un absurdo, pero es así. La literatura es un vicio basado en la vanidad. El periodismo es una utilidad pública. Un periodista puede ser buena persona. Un literato es casi siempre un bellaco disfrazado. (Matus párr. 23)

Junto a Edwards Bello, Álvaro Matus destaca a «Carlos Silva Vildósola, quien fuera director de El Mercurio; el satírico Joaquín Díaz Garcés, que firmaba Ángel Pino; Genaro Prieto, un conservador con mucho sentido del humor [...] Mario Rivas, sarcástico columnista de Las Noticias Gráficas que bautizó a Benjamín Subercaseaux como Benjamona Subercasiútica» (párr. 25).

El caso de Joaquín Díaz Garcés refleja con meridiana claridad la evolución de la crónica centrada en la crítica a la modernidad a la crítica explícitamente política. Así, por ejemplo, en el período de la primera década del siglo $X X$, se detiene en temáticas que disectan irónicamente el acomodamiento de la cultura chilena al contexto cada vez más abierto que le ofrece el concierto internacional. Sus columnas sobre la identidad chilena («La chicha», «La sandía», «La cafetera rusa», «Matrimonio con príncipe», «Psicología del intruso»), sobre los avances dela modernización a la criolla («Interiores modernos», «Reformas y progresos médicos», «Huevos importados»), sobre las nostalgias del orden que desaparece 
(«Hombres de ayer y de hoy», «Una figura de antaño»), etc., dan cuenta de ello. Sin embargo, el tono irónico y elegante con que enfrenta dichas cuestiones da paso, sobre todo hacia fines de la segunda década, a una crítica abiertamente sarcástica, menos aguda e ingeniosa, abocada principalmente a criticar explícitamente el proyecto político levantado en Chile por el candidato progresista y posterior presidente de la república, Arturo Alessandri Palma. Este cambio temático y de estilo da cuenta, en el contexto de la provinciana realidad chilena, del proceso que, según vimos, se estaba dando en todo el subcontinente latinoamericano, es decir, el paso de la ciudad modernizada a la ciudad politizada.

\section{Mario Vargas Llosa y Gabriela Mistral, dos casos emblemáticos}

He escogido estos dos ejemplos para ilustrar, en el contexto latinoamericano, la presencia de dos escritores que marcaron la escena literaria del siglo XX. Ambos, gracias a su producción y reconocimiento, recibieron el Premio Nobel; ambos colaboraron en la prensa (Vargas Llosa lo sigue haciendo) y cumplen con ofrecer un ejemplo de cómo el ethos retórico se constituye en la condición comunicativa entre escritor y lector. En el caso de Mario Vargas Llosa, su autoridad como novelista es indiscutida, pero, en el momento en que ingresó en el mundo de la «opinión», ya no fueron sus cualidades narrativas las que entraron en juego, sino el grado de compromiso ideológico con que inscribió su participación en los distintos medios, pesándole muy en contra la derechización de su pensamiento, sobre todo cuando, en los años del boom, había destacado junto a varios de sus coetáneos (García Márquez) por planteamientos bastante progresistas. Hace algunas décadas, leí en un periódico santiaguino (Chile) que él había renunciado a ser el Flaubert de las letras hispanoamericanas para convertirse en el Julio Iglesias de la política peruana. Sin embargo, ¡qué duda cabe! su calidad intelectual ha logrado superar ese primer momento y lo vemos actualmente, sumado además el prestigio del Premio Nobel de Literatura, como un opinionista al que, pese a nuestras diferencias ideológicas, no se puede dejar de consultar e, incluso, de disfrutar. Tal es el caso de sus colaboraciones con el periódico peruano La República, en donde podemos encontrar reflexiones sobre las últimas elecciones europeas y la declinación del orden occidental, sobre la abdicación del Rey Juan Carlos I y el estado 
actual de la monarquía, la participación de los estudiantes en las revueltas en contra del movimiento chavista que gobierna ahora en Venezuela a través de su heredero Nicolás Maduro, el proyecto de ley para uniones civiles de homosexuales en Perú y las resistencias arcaicas de los sectores tradicionales y de la Iglesia Católica, reflexiones sobre la obra de JeanFrancois Revel revitalizada con la monumental biografía de Philippe Boulanger,etc. En todosestos casos, se deja entrever lafascinaciónescritural y la autoridad intelectual de un escritor que, superados sus flirteos con la política de su país de origen, logra refrescar tópicos trascendentes que refuerzan su vocación liberal en contra de los nacionalismos, el racismo, la heteronormatividad, la barbarie que subsiste en la mayor parte de los órdenes políticos nacionales, y el trasnochado discurso de algunos países anclados aún en utopías sesentistas. No obstante, el vaivén de su progresismo a veces tropieza con ciertas fidelidades que cuesta entender, como es el caso de una ausencia crítica frente a la monarquía española y la necesidad de repensar un orden constitucional que no dependa de la sucesión de sangre para atribuir responsabilidades y derechos políticos, pero todos sabemos que la autonomía se pierde cuando hay deudas que pagar, sobre todo tratándose del Marqués de Vargas Llosa.

Otro ejemplo interesante de consignar, de data más antigua, es el de Gabriela Mistral, poeta chilena que estudió en una humilde escuela campesina, que no tuvo ninguno de los privilegios de cuna de la mayor parte de sus coetáneos poetas e intelectuales y que, desde la más recóndita provincia, se instaló en el escenario americano como un referente fundamental a la hora de fundar una visión americanista. Su forma de participar en la prensa es aleatoria y variada, pero fue el concurso de sus numerosas intervenciones extraliterarias la que creó en torno a ella ese mito de «madre americana», una especie de «mama grande» garcimarquiana, arrastrando su manto de dolorosa y poderosa matriarca desde México hasta Tierra del Fuego y cubriendo con su protección la memoria indígena, el niño de pies descalzos, la mujer campesina. La mayor parte de sus colaboraciones, varias de ellas en diversos periódicos, otras como cartas o «recados», son recogidas en la revista costarricense Repertorio Americano (1919-1958).

Es interesante observar que, en la incursión en el periódico, estos destacados escritores dan cuenta de la exigencia que el periodismo 
impone al escritor, al someterlo a la emergencia del día a día con sus temáticas variadas, tal como lo hacía notar Manuel Gutiérrez Nájera en 1883, en su crónica «Su majestad el periodista»:

Ayer fue economista, hoy es teólogo, mañana será hebraizante o tahonero. Es necesario que sepa cómo se hace el buen pan y cuáles son las leyes de la evolución; no hay ciencia que no esté obligado a conocer, ni arte cuyos secretos deban ser ignorados por su entendimiento; la misma pluma con que anoche dibujó la crónica del baile o del teatro le servirá para trazar hoy un artículo sobre ferrocarriles o sobre bancos, y todo eso sin que la premura del tiempo le permita abrir un libro o consultar un diccionario. (ctd. por González 80)

Y es que en ello se advierte la dinámica vertiginosa de la modernidad, el tiempo acuciante, la rapidez de la máquina, la versatilidad de las noticias que llegan a través del telégrafo, de las cuales no puede abstraerse el escritor si quiere establecer, en sus artículos de opinión, una conexión con los lectores de su tiempo. Así pues, Gabriela Mistral y Mario Vargas Llosa son ejemplo de que la vigencia ética de su ejercicio periodístico es también compromiso con su circunstancia vital.

\section{El ethos retórico y la coherencia del «yo»}

Aníbal González (1983), al comentar el trasplante del género de la chronique a Hispanoamérica, atribuye un rol fundamental al mexicano Manuel Gutiérrez Nájera, y al cubano José Martí. «Podríamos decir que a Nájera le toca introducir el género y a Martí el darle mayor relieve intelectual y difusión continental» (77). Con respecto a Martí, González problematiza un aspecto que ha de ser fundamental para la comprensión de la vinculación entre escritores y prensa; a saber: la coherencia del yo escritural, es decir, el modo en que, variando de asunto, de objeto y de contexto cada día, el autor logra mostrarse el mismo sujeto enunciante en todas sus columnas. Este dilema no fue menor para Martí, pues, formado en la tradición ilustrada, la que concebía al escritor como autor de obras de gran aliento, esta imposición moderna de escribir crónicas sobre temas variados en una sucesión que podía parecer caótica, constituía una amenaza y un desafío exigentes. En carta a Bartolomé Mitre, dice: 
Es mal mío no poder concebir nada en retazos, y querer cargar de esencia los pequeños moldes, y hacer los artículos de diario como si fueran libros, por lo cual no escribo con sosiego, ni con mi verdadero modo de escribir, sino cuando siento que escribo para gentes que han de amarme, y cuando puedo, en pequeñas obras sucesivas, ir contorneando insensiblemente en lo exterior la obra previa hecha para mí. (Citado por González 79)

La preocupación de Martí puede resultar extraña para nosotros, pero no para él, no para su tiempo, en que las nuevas relaciones entre el escritor y el periodismo estaban en plena etapa de reconfiguración. Se agudiza más aun en su caso, tratándose de un escritor que "disciplinó y dio su vida al servicio de un ideal» (González 79), es decir, de quien necesitaba que toda su producción escritural estuviera al servicio inconfundible de un mismo derrotero y de los valores que esperaba relevar. El desafío fue superado, pues, tal como indica González, luchando «con las armas de la filología y el estilo», «[p]or sobre la diversidad de temas y enfoques a que lo obligaba la crónica, Martí se propuso imponer la coherencia de su «yo» y de una voz poética potente, uniforme y original» (79). Igual dificultad experimentó Nájera, para quien «la labor periodística significaba un desmembramiento [...] del cronista», a diferencia del escritor que puede «intentar aquella maciza labor de síntesis enciclopédica tan típica de la prosa decimonónica, de Hegel a Renan, y de Balzac a Zola» (González 80). En este proceso, el enemigo principal es el tiempo, la urgencia de responder con la crónica cotidiana antes del cierre de edición; variedad y rapidez, exigencias de la vida y el periodismo modernos que impelen al escritor que, en búsqueda de un sustento económico, se ve amenazado en su integridad autorial.

Esta pregunta por la coherencia del yo alude a una dimensión central en la valoración de la autoridad del escritor. Después de un siglo y medio de relaciones con la prensa, nos resulta muy útil para distinguir y elevar por sobre el horizonte la obra de los grandes columnistas, cronistas, opinionistas. Puede que no recordemos en particular una $\mathrm{u}$ otra crónica de Miguel de Unamuno, pero no nos resulta indiferente su identidad hispánica hecha de dolor, de inconformidad y de irreverencia. En esa misma orientación, distinguimos el color magistral, materno, americanista de las crónicas mistralianas, así como el universalismo, la 
erudición y la actualidad de las columnas de Vargas Llosa. Y, así como Sarmiento podía decir, en una carta que escribió a Paul Groussac, en 1887, «En español nada hay que se parezca a la salida de bramidos de Martí, y después de Víctor Hugo nada presenta la Francia de esa resonancia de metal» (ctd. por González 79), también nosotros podemos recoger la coherencia retórica y moral de los escritores que le siguieron.

\section{Conclusiones}

La relación entre escritores y prensa se problematiza desde el surgimiento del periodismo moderno, el cual genera nuevas condiciones de participación en la escena pública para los primeros. Dichas condiciones están determinadas por varios factores. En primer lugar, el escritor de fines del siglo XIX se sitúa en un lugar distinto del que ocuparon los intelectuales fundadores de la prensa latinoamericana. En segundo lugar, la prensa moderna se caracteriza por su valoración de la «verdad objetiva» fielmente representada por el desarrollo de la noticia, y el reportaje, como géneros destinados al ejercicio de un nuevo tipo de profesional, el periodista. En medio de la noticia, se genera un espacio para el comentario, la crónica, la columna de opinión (en palabras de nuestros días); son estos los géneros en los que incursionará el escritor moderno. Desde ellos emanará su discurso a menudo crítico, propositivo e, incluso, alternativo, reflejo de su nuevo posicionamiento en el campo cultural.

Los diversos géneros ligados a la columna cultural someterán a prueba la relación entre los conceptos de «autoría»y «autoridad». La autoría, como evidencia del buen nombre adquirido por el escritor gracias a sus obras y su autoridad como valoración subjetiva del capital social con que cuenta. Hubo autores que, en el concurso de su participación en la prensa, hicieron valer su buen nombre y crecieron en autoridad y prestigio, como es el caso de Emile Zola, José Martí y Gabriela Mistral. Ha habido otros que han vivido desplazamientos zigzagueantes en esta materia, así, por ejemplo, Mario Vargas Llosa.

Lo que, en definitiva, resuelve la cuestión de la autoridad literaria está dada por dos factores fundamentales. En primer lugar, el estilo, es decir, las cualidades retóricas que definen y caracterizan la escritura de un columnista, y, en segundo lugar, la competencia moral reconocida 
socialmente para referirse con propiedad sobre cuestiones determinadas. Esto es lo que, siguiendo a López Pan, se llama ethos retórico. Estilo sin respaldo ético no convence, así como un compromiso moral potente sin estilo literario no seduce. Ambos componentes son la clave de análisis para validar la autoridad de un escritor determinado en la prensa. Y son, finalmente, la condición que garantiza el salvamento del «yo» autorial, es decir, la integridad amenazada por la naturaleza fragmentaria de los subgéneros relacionados con la columna cultural.

\section{Referencias bibliográficas}

González, Aníbal. La crónica modernista hispanoamericana. Madrid, Porrúa S. A. 1983.

La Prosa Modernista. «Francisco García Cisneros». Prosa Modernista. En línea http://www.prosamodernista.com/prosa-premodernista/ francisco-garcia-cisneros

López Pan, Fernando. «El ethos retórico, un rasgo común a todas las modalidades del género columna». Ínsula, julio-agosto, $\mathrm{n}^{\circ} 703$ 704, 2005, pp. 12-15.

Martí, José. «Prólogo al poema del Niágara». En Obras completas. La Habana, 1975 [1882].

Matus, Álvaro. «Historia abreviada del periodismo de opinión». Revista Dossier, no. 2, web. http://www.revistadossier.cl/tema-historiaabreviada-del-periodismo-de-opinion-alvaro-matus /

Rama, Ángel. La ciudad letrada. Montevideo, Arca, 1998 [1984].

Subercaseaux, Bernardo. Genealogía de la vanguardia. Santiago, Ediciones Universidad de Chile, 1997. 